\title{
Folding of a 2-Tangle-Graphs
}

\author{
M. El-Ghoul \\ Mathematics Department, Faculty of Science, Tanta University, Tanta, Egypt \\ E-mail: melghoul2008@yahoo.com \\ F. Salama \\ Mathematics Department, Faculty of Science, Tanta University,Tanta, Egypt \\ E-mail: Fatma2salama@yahoo.com
}

A. S. Pelila

Mathematics Department, Faculty of Science, Tanta University,Tanta, Egypt

Received: May 23, 2011 Accepted: June 9, 2011 Published: February 1, 2012

doi:10.5539/jmr.v4n1p86 URL: http://dx.doi.org/10.5539/jmr.v4n1p86

\begin{abstract}
In this paper, we will define foldings of 2-tangle-graph into itself. The representation of this new graph and its foldings by matrices will be presented. We will deduce the limit of foldings of this new graph.
\end{abstract}

Keywords: Graphs, Tangles, Folding

2000 Mathematics Subject Classification: 51H10, 57N20

\section{Definitions and Background}

There are many physical systems whose performance depends not only on the characteristics of the components but also on the relative locations of the elements. An obvious example is an electrical network. If we change a resistor to a capacitor, generally some of the properties (such as an input impedance of the network) also change. This indicates that the performance of a system depends on the characteristics of the components. If, on the other hand, we change the location of one resistor, the input impedance again may change, which shows that the topology of the system is influencing the system's performance. There are systems constructed of only one kind of component so that the system's performance depends only an its topology. An example of such a system is a single-contact switching circuit. Similar situations can be seen is non physical systems such as structures of administration. Hence it is important to represent a system so that its topology can be visualized clearly.

On simple way of displaying structure of a system is to draw a diagram consisting of points called vertices and line segments called edges which connected these vertices so that such vertices and edges indicate components and relationships between these components. Such a diagram is called a linear graph whose name depends on the kind of physical system we deal with. This means that it may be called a network, a net, a circuit, a graph, a diagram a structure, and so on.

Definition 1 An abstract graph $G$ is a diagram consisting of finite non empty set of elements called vertices denoted by $V(G)$ together with a set of unordered pairs of these elements called edges denoted by $E(G)$. The set of vertices of the graph $G$ is called the vertex-set of $G$ and the list of the edges is called the edge-list of $G$ (Beineke \& Wilson, 1983; El-Ghoul, 1993; White, 1973; Wilson, 1972).

Definition 2 Let $v$ and $w$ be two vertices of a graph. If $v$ and $w$ are joined by an edges, then $v$ and $w$ are said to be adjacent. Also, $v$ and $w$ are said to be incident with e then e is said to be incident with $v$ and $w$ (Wilson \& Watkins, 1990).

Definition 3 Let $G$ be a graph without loops, with $n$-vertices labeled $1,2,3, \ldots, n$. The "adjacency matrix" $A(G)$ is the $n \times n$ matrix in which the entry in row $i$ and column $j$ is the number of edges joining the vertices $i$ and $j$ (Wilson $\&$ Watkins, 1990).

Definition 4 Let $G$ be a graph without loops, with $n$-vertices labeled $1,2,3, \ldots, n$ and $m$ edges labeled $1,2,3, \ldots, m$. The incidence matrix $I(G)$ is the $n \times m$ matrix in which the entry in row $i$ and column $j$ is 1 if vertex i is incident with edge $j$ and 0 otherwise (Wilson \& Watkins, 1990).

Example 1 Consider the graph $G$ in Figure 1. 
$<$ Figure 1 $>$

The adjacency matrix $A(G)$ is $A(G)=\left[\begin{array}{lll}0 & 1 & 1 \\ 1 & 0 & 2 \\ 1 & 2 & 0\end{array}\right]$ and the incidence matrix of $G$ is $I(G)=\left[\begin{array}{cccc}1 & 0 & 0 & 1 \\ 1 & 1 & 1 & 0 \\ 0 & 1 & 1 & 1\end{array}\right]$.

Definition 5 Let $D$ be a unite cube with n points $a_{1}, a_{2}, \ldots, a_{n}$ on the top face and $n$ points $b_{1}, b_{2}, \ldots, b_{n}$ on the bottom face. A set of n mutually disjoint simple $\operatorname{arcs} d_{1}, d_{2}, \ldots, d_{n}$ in $D$ that connect $a_{1}, a_{2}, \ldots, a_{n}$ to $b_{j 1}, b_{j 2}, \ldots, b_{j n}$ respectively, is called an $(n, n)$-tangle, where $\left(b_{j 1}, b_{j 2}, \ldots, b_{j n}\right)$ is a permutation of $(1,2, \ldots)$ in Fig. 2 (a) is $(1,1)$-tangle and Fig. 2 (b) is (2,2)-tangle (Marasugi, \& Kurpita, 1999).

$<$ Figure $2>$

Definition 6 Let $D$ be a unit cube, so $D=\{(x, y, z): 0 \leq x, y, z \leq 1\}$ on the top face of cube place n points, $a_{1}, a_{2}, \ldots, a_{n}$ and similarly place $\mathrm{n}$ points on the bottom face $b_{1}, b_{2}, \ldots, b_{n}$. Now join the points $a_{1}, a_{2}, \ldots, a_{n}$ with $b_{1}, b_{2}, \ldots, b_{n}$ by means of $\mathrm{n} \operatorname{arcs} d_{1}, d_{2}, \ldots, d_{n}$ (as smooth curves), this arcs are mutually disjoint and each $d_{I}$ connects some $a_{J}$ to $b_{K}$ (J=K or $J \neq K$ ) not connected $a_{J}$ to $a_{K}$ or $a_{J}$ to $b_{K}$. Each plane $E_{s}$, such that $z=s, 0 \leq s \leq 1$ (parallel to $x y$-plane), intersections each $\operatorname{arc} d_{i}$ at one and only point. A configuration of $\mathrm{n} \operatorname{arcs} d_{1}, d_{2}, \ldots, d_{n}$ with end points $a_{1}, a_{2}, \ldots, a_{n}$ and $b_{1}, b_{2}, \ldots, b_{n}$ is called $\mathrm{n}$-braid or a braid with $\mathrm{n}$ strings denoted by $\beta_{n}$, see Figure 3 (Marasugi, \& Kurpita, 1999).

$<$ Figure $3>$

Definition 7 Let $f: G \rightarrow G^{\prime}$ be a map between any two graphs $G$ and $G^{\prime}$ (not necessary to be simple) such that if $(u, v) \in G,(f(u), f(v)) \in G^{\prime}$, then $f$ is called a "topological folding" of $G$ to $G^{\prime}$ provided that $d(f(u), f(v)) \leq d(u, v)$ (El-Ghoul, \& Homoda, 2006).

Definition 8 Let $g: G \rightarrow G^{\prime}$ be a map between any two graphs $G$ and $G^{\prime}$ (not necessary to be simple) such that if $(u, v) \in G,(g(u), g(v)) \in G^{\prime}$, then $g$ is called a "topological unfolding" of $G$ to $G^{\prime}$ provided that $d(g(u), g(v))>d(u, v)$ (El-Ghoul, \& Homoda, 2006).

\section{The Main Results}

Definition 1 A general 2-tangle-graph is an abstract graph in which each edge is a tangle in 2-dimension and the type of tangles is different and each vertex is a finite non empty set of vertices of an abstract graph. i.e. $G_{t}(V, E)=$ $\left(\left\{\left\{v_{11}, v_{12}, v_{13}, \ldots, v_{1 n}\right\},\left\{v_{21}, v_{22}, \ldots, v_{2 m}\right\},\left\{v_{31}, v_{32}, \ldots, v_{3 l}\right\}, \ldots\left\{v_{n 1}, v_{n 2}, \ldots, v_{n k}\right\}\right\} .\left\{\left\{e_{11}, e_{12}, \ldots, e_{1 n-1}\right\},\left\{e_{21}, e_{22}, \ldots, e_{2 m-1}\right\}\right.\right.$, $\left.\left.\ldots,\left\{e_{n-11}, e_{n-12}, \ldots, e_{n-1 k-1}\right\}\right\}\right)$.

\subsection{Folding of a 2-tangle-graph}

Such that in which each edge is disc, Figure 4.

$<$ Figure $4>$

The adjacency and incidence matrices of $G_{t}$ given by

$$
A\left(G_{t}\right)=\left[\begin{array}{ccc}
0 & 1_{1 s^{2}} & 0 \\
1_{1 s^{2}} & 0 & 1_{1 s^{2}} 1_{1 s^{2}} \\
0 & 1_{1 s^{2}} 1_{1 s^{2}} & 0
\end{array}\right], I\left(G_{t}\right)=\left[\begin{array}{cc}
1_{1 s^{2}} & 0 \\
1_{1 s^{2}} & 1_{1 s^{2}} \\
0 & 1_{1 s^{2}}
\end{array}\right] .
$$

Case 1 The folding of tangle-vertex to its 1-neighbourhood tangle-vertex such folding $V_{1}$ to $V_{2}$ in FIGURE 4 , we obtain a tangle-graph $G_{t}$ as shown in Figure 5.

$<$ Figure 5>

The adjacency and incidence matrices of $\mathrm{G}_{t}$ given by

$$
\begin{gathered}
A\left(G_{t}^{\prime}\right)=\left[\begin{array}{cc}
1_{1 s^{2} 1 s^{2}}^{102^{2}} & 1_{1 s^{2} 1 s^{2}} \\
1_{1 s^{2} 1 s^{2}} & 0
\end{array}\right], I\left(G_{t}^{\prime}\right)=\left[\begin{array}{c}
1_{1 s^{2} 1 s^{2}}^{1^{2}} \\
1_{1 s^{2}} 1_{1 s^{2}}
\end{array}\right], \\
A\left(G_{t}^{\prime \prime}\right)=\left[1^{1^{0} 2^{2} 1^{2} 2^{1}}\right], I\left(G_{t}^{\prime}\right)=[0] .
\end{gathered}
$$

Proposition 1 The graph which results from folding of tangle vertex to its 1-neighbourhood tangle vertex in a tangle graph is a tangle graph with loop.

Proposition 2 The limit of foldings of 2-tangle vertex into its 1-neighbourhood tangle vertex in a 2-tangle-graph is a null 2-tangle graph with loops.

Case 2 The folding of tangle-vertex to anther tangle-vertex which is not 1- neighbourhood to it, see Figure 6. 
$<$ Figure 6>

The adjacency and incidence matrices of $G_{t}, G_{t}^{\prime}, G^{\prime \prime}{ }_{t}$ given by

$$
\begin{gathered}
A\left(G_{t}^{\prime}\right)=\left[\begin{array}{cc}
0 & 1_{1 s^{2} 1 s^{2}} \\
1_{1 s^{2} 1 s^{2}} & 0
\end{array}\right], I\left(G_{t}^{\prime}\right)=\left[\begin{array}{l}
1_{1 s^{2} 1 s^{2}} \\
1_{1 s^{2} 1 s^{2}}
\end{array}\right], \\
A\left(G_{t}^{\prime \prime}\right)=\left[\begin{array}{cc}
0 & 1_{1 s^{2} 1 s^{2}} 1_{1 s^{2}} \\
1_{1 s^{2} 1 s^{2}} 1_{1 s^{2}} & 0
\end{array}\right], I\left(G_{t}^{\prime}\right)=\left[\begin{array}{ll}
1_{1 s^{2} 1 s^{2}} & 1_{1 s^{2}} \\
1_{1 s^{2} 1 s^{2}} & 1_{1 s^{2}}
\end{array}\right] .
\end{gathered}
$$

Proposition 3 The foldings of tangle-vertex of a 2-tangle-graph without loop is a 2-tangle graph without loop too.

Proposition 4 The limit of foldings of a tangle-vertex to other tangle-vertex which is not 1-neighbourhood vertex in a 2-tangle-graph is a 2-tangle-graph with two tangle-vertex.

Case 3 Folding of termenal tangle-vertices see Figure 7.

$<$ Figure $7>$

The adjacency and incidence matrices of $\mathrm{G}_{t}$ given by

$$
A\left(G_{t}^{\prime}\right)=\left[\begin{array}{ccc}
0 & 1_{1 s^{1}} & 1_{1 s^{1} 1 s^{1} 1 s^{1}} \\
1_{1 s^{1}} & 0 & 1_{1 s^{1} 1 s^{1}} \\
1_{1 s^{1} 1 s^{1} 1 s^{1}} & 1_{1 s^{1} 1 s^{1}} & 0
\end{array}\right], I\left(G_{t}^{\prime}\right)=\left[\begin{array}{ccc}
1_{1 s^{1}} & 0 & 1_{1 s^{1} 1 s^{1} 1 s^{1}} \\
1_{1 s^{1}} & 1_{1 s^{1} 1 s^{1}} & 0 \\
0 & 1_{1 s^{1} 1 s^{1}} & 1_{1 s^{1} 1 s^{1} 1 s^{1}}
\end{array}\right] .
$$

Proposition 5 The folding of terminal tangle-vertices of open 2-tangle-graph is a closed 2-tangle-graph.

Case 4 Folding of a tangle-edge to another tangle-edge, see Figure 8.

$<$ Figure 8>

$$
A\left(G_{t}^{\prime}\right)=\left[\begin{array}{cc}
0 & 1_{1 s^{2} 1 s^{2}} \\
1_{1 s^{2} 1 s^{2}} & 0
\end{array}\right], I\left(G_{t}^{\prime}\right)=\left[\begin{array}{c}
1_{1 s^{2} 1 s^{2}} \\
1_{1 s^{2} 1 s^{2}}
\end{array}\right] .
$$

Proposition 6 The foldings of a tangle-edge to its 1-neighbourhood tangle-edge in a 2-tangle-graph is 2-tangle-graph with the number of edges decrease the tangle-edges in a 2-tangle-graph by 1 .

Proposition 7 The limit of foldings of a tangle edge to its 1-neighbourhood tangle edge in a 2-tangle-graph is 2-tangle graph with two vertices.

Case 5 Folding of tangle edge to an edge which is not 1-neighbourhood tangle edge, see Figure 9.

$<$ Figure 9>

The adjacency and incidence matrices of $G_{t}$ given by

$$
A\left(G_{t}^{\prime}\right)=\left[\begin{array}{cc}
1^{1^{0} 2^{3} 3^{2}} & 1_{1 s^{1} 1 s^{1} s^{1}} \\
1_{1 s^{1} 1 s^{1} 1 s^{1}} & 0
\end{array}\right], I\left(G_{t}^{\prime}\right)=\left[\begin{array}{c}
1_{1}^{1^{0} 2^{3} 3^{2}} \\
1_{1 s^{1} 1 s^{1} 1 s^{1}}
\end{array}\right] .
$$

Proposition 8 The folding of a tangle-edge to other tangle-edge in a 2-tangle-graph with loop.

Proposition 9 The limit of foldings of a tangle-edge to other tangle-edge in a 1-tangle-graph is a 2-tangle-graph with two vertices and loops.

Case 6 Folding of a tangle-edge into itself, see Figure 10.

$<$ Figure 10>

Proposition 10 The limit of foldings of a tangle-edge into itself in a 2-tangle-graph is a 2-tangle-graph decrease the number of tangle -vertices and tangle-edge by 1 .

Proposition 11 The end of limit of foldings of an edge of a 2-tangle-graph into itself is a 2-tangle-vertex which has the largest number of vertices.

Case 7 Folding of all edges into themselves, see Figure 11.

$<$ Figure 11>

Proposition 12 The limit of foldings of all edges to themselves in a tangle-graph is a null tangle-graph has only one tangle vertex. 
Proposition 13 The limit of foldings of a tangle-edges into themselves in a 2-tangle-graph is equivalent to the end of limit of folding of a tangle-edge into itself.

Case 8 Folding of an inner vertices into themselves, see Figure 12.

$<$ Figure 12>

Notation 1 The graph $G_{t}^{\prime \prime \prime \prime}$ is not tangle-graph.

Case 9 Folding of an inner edges into another inner edge, see Figure 13.

$<$ Figure 13>

Case 10 Folding of each inner edge into itself, see Figure 14.

$<$ Figure 14 $>$

Proposition 14 The limit of foldings of each inner edges into itself in a 2-tangle-graph is a linear 1-tangle-graph.

2.2 A general 2-tangle-graph in which each edge is a sheet and each vertex is a segment

As shown in Figure 15.

$<$ Figure 15>

The adjacency and incidence matrices given by

$$
\begin{gathered}
A\left(G_{t}\right)=\left[\begin{array}{cccc}
0 & 1_{1 r^{2}} & 0 & 0 \\
1_{1 r^{2}} & 0 & 1_{1 r^{2} 1 r^{2}} & 0 \\
0 & 1_{1 r^{2} 1 r^{2}} & 0 & 1_{1 r^{2} 1 r^{2} 1 r^{2}} \\
0 & 0 & 1_{1 r^{2} 1 r^{2} 1 r^{2}} & 0
\end{array}\right], \\
I\left(G_{t}\right)=\left[\begin{array}{ccc}
1_{1 r^{2}} & 0 & 0 \\
1_{1 r^{2}} & 1_{1 r^{2} 1 r^{2}} & 0 \\
0 & 1_{1 r^{2} 1 r^{2}} & 1_{1 r^{2} 1 r^{2} 1 r^{2}} \\
0 & 0 & 1_{1 r^{2} 1 r^{2} 1 r^{2}}
\end{array}\right]
\end{gathered}
$$

Case 11 The folding of tangle-vertex to its 1-neighbourhood tangle-vertex such folding $V_{1}$ to $V_{2}$ in FIGURE 14 , we obtain a tangle-graph $G_{t}$ in Figure 16.

$<$ Figure 16>

The adjacency and incidence matrices of $G_{t}$ given by

$$
\begin{gathered}
A\left(G_{t}^{\prime}\right)=\left[\begin{array}{ccc}
1^{1^{0} 2^{2}} & 1_{1 r^{2} 1 r^{2}} & 0 \\
1_{1 r^{2} 1 r^{2}} & 0 & 1_{1 r^{2} 1 r^{2} 1 r^{2}} \\
0 & 1_{1 r^{2} 1 r^{2} 1 r^{2}} & 0
\end{array}\right], I\left(G_{t}^{\prime}\right)=\left[\begin{array}{cc}
1_{1 r^{2} 1 r^{2}}^{1^{2}} & 0 \\
1_{1 r^{2} 1 r^{2}} & 1_{1 r^{2} 1 r^{2} 1 r^{2}} \\
0 & 1_{1 r^{2} 1 r^{2} 1 r^{2}}
\end{array}\right] \\
A\left(G_{t}^{\prime \prime}\right)=\left[\begin{array}{cc}
1_{1 r^{2} 1 r^{2} 1 r^{2}}^{1^{2}} & 1_{1 r^{2} 1 r^{2} 1 r^{2}} \\
1_{1 r^{2} 1 r^{2} 1 r^{2}} & 0
\end{array}\right], I\left(G_{t}^{\prime \prime}\right)=\left[\begin{array}{c}
1_{1 r^{2} 1 r^{2} 1 r^{2}}^{1^{2}} \\
1_{1 r^{2} 1 r^{2} 1 r^{2}}
\end{array}\right] \\
A\left(G_{t}^{\prime \prime \prime}\right)=\left[1^{1^{0} 2^{2} 1^{2} 2^{1} 1^{1} 2^{3} 3^{2}}\right], I\left(G_{t}^{\prime \prime \prime}\right)=[0] .
\end{gathered}
$$

Proposition 15 The graph results from folding of tangle vertex to its 1-neighbourhood tangle vertex in a tangle graph is a tangle graph with loop.

Proposition 16 The limit of foldings of 2-tangle vertex into its 1-neighbourhood tangle vertex in a 2-tangle-graph is a null 2-tangle graph with loops.

Case 12 The folding of tangle-vertex to anther tangle-vertex which is not 1-neighbourhood to it, see Figure 17.

$<$ Figure $17>$

The adjacency and incidence matrices of $G_{t}, G_{t}^{\prime}, G_{t}^{\prime \prime}$ given by

$$
A\left(G_{t}^{\prime}\right)=\left[\begin{array}{ccc}
0 & 1_{1_{r^{2}} 1_{r^{2}}} & 0 \\
1_{1_{r^{2}} 1_{r^{2}}} & 0 & 1_{1_{r^{2}} 1_{r^{2}} 1_{r^{2}}} \\
0 & 1_{1_{r^{2}} 1_{r^{2}} 1_{r^{2}}} & 0
\end{array}\right], I\left(G_{t}^{\prime}\right)=\left[\begin{array}{cc}
1_{1_{r^{2}} 1_{r^{2}}} & 0 \\
1_{1_{r^{2}} 1_{r^{2}}} & 1_{1_{r^{2}} 1_{r^{2}} 1_{r^{2}}} \\
0 & 1_{1_{r^{2}} 1_{r^{2}} 1_{r^{2}}}
\end{array}\right],
$$




$$
\begin{aligned}
& A\left(G_{t}^{\prime \prime}\right)=\left[\begin{array}{ccc}
0 & 1_{1_{r^{2}} 1_{r^{2}}} 1_{1_{r^{2}}} & 0 \\
1_{1_{r^{2}} 1_{r^{2}}} 1_{1_{r^{2}}} & 0 & 1_{1_{r^{2}} 1_{r^{2}} 1_{r^{2}}} \\
0 & 1_{1_{r^{2}} 1_{r^{2}} 1_{r^{2}}} & 0
\end{array}\right], \\
& I\left(G_{t}^{\prime \prime}\right)=\left[\begin{array}{ccc}
1_{1_{r^{2}}} & 1_{1_{r^{2}} 1_{r^{2}}} & 0 \\
1_{r_{r 2}} & 1_{r_{r^{2}} r_{r^{2}}} & 1_{r_{r^{2}} 1_{r^{2}} 1_{r^{2}}} \\
0 & 0 & 1_{r_{r^{2}} 1_{r^{2}} 1_{r^{2}}}
\end{array}\right] \text {. }
\end{aligned}
$$

Proposition 17 The foldings of tangle-vertex of a sheeted 2-tangle-graph without loop is a sheeted 2-tangle graph with out loop too.

Proposition 18 The limit of foldings of a tangle-vertex to other tangle-vertex which is not 1-neighbourhood vertex to it in a sheeted 2-tangle-graph is a sheeted 2-tangle-graph with two tangle-vertex.

Case 13 The folding of termenal tangle-vertices, see Figure 18.

$<$ Figure 18>

The adjacency and incidence matrices of $G_{t}$ given by

$$
\begin{aligned}
& A\left(G_{t}\right)=\left[\begin{array}{ccc}
0 & 1_{1, r^{2}} & 1_{1_{r^{2}} 1_{r^{2}} 1_{r^{2}}} \\
1_{1_{r^{2}}} & 0 & 1_{r_{r^{2}} 1_{r^{2}}} \\
1_{r_{r 2} 1_{r^{2}} 1_{r^{2}}} & 1_{1_{r^{2}} 1_{r^{2}}} & 0
\end{array}\right], \\
& I\left(G_{t}\right)=\left[\begin{array}{ccc}
1_{1_{r^{2}}} & 0 & 1_{1_{r^{2}} 1_{r^{2}} 1_{r^{2}}} \\
1_{r_{r 2}} & 1_{r_{r^{2}} 1_{r^{2}}} & 0 \\
0 & 1_{r_{r^{2}} 1_{r^{2}}} & 1_{1_{r^{2}} 1_{r^{2}} 1_{r^{2}}}
\end{array}\right] .
\end{aligned}
$$

Proposition 19 The folding of terminal tangle-vertices of open sheeted 2-tangle-graph is a closed sheeted 2-tangle-graph.

Case 14 The folding of a tangle-edge to 1-neighbourhood tangle-edge, see Figure 19.

$<$ Figure 19>

$$
\begin{aligned}
& A\left(G_{t}^{\prime}\right)=\left[\begin{array}{ccc}
0 & 1_{1_{r^{2}} 1_{r^{2}}} & 0 \\
1_{1_{r 2} 1_{r^{2}}} & 0 & 1_{r_{r^{2}} 1_{r_{r}} 1_{r^{2}}} \\
0 & 1_{1_{r^{2}} 1_{r^{2}}} & 0
\end{array}\right], I\left(G_{t}^{\prime}\right)=\left[\begin{array}{cc}
1_{1_{r} 1_{r^{2}}} & 0 \\
1_{1_{r^{2}} 1_{r^{2}}} & 1_{1_{r^{2}} 1_{r^{2}} 1_{r^{2}}} \\
0 & 1_{1_{r^{2}} 1_{r^{2}} 1_{r^{2}}}
\end{array}\right] . \\
& A\left(G_{t}^{\prime \prime}\right)=\left[\begin{array}{cc}
0 & 1_{1_{r^{2}} 1_{r^{2}} 1_{r^{2}}} \\
1_{r_{r^{2}} 1_{r^{2}} 1_{r^{2}}} & 0
\end{array}\right], I\left(G_{t}^{\prime \prime}\right)=\left[\begin{array}{c}
1_{1_{r^{2}} 1_{r^{2}} 1_{r^{2}}} \\
1_{r_{r^{2}} 1_{r^{2}} 1_{r^{2}}}
\end{array}\right] \text {. }
\end{aligned}
$$

Proposition 20 The foldings of a tangle-edge to its 1-neighbourhood tangle-edge in a sheeted 2-tangle-graph is 2-tanglegraph with the number of edges decrease the tangle-edges in a sheeted 2-tangle-graph by 1 .

Proposition 21 The limit of foldings of a tangle edge to its 1-neighbourhood tangle edge in a sheeted 2-tangle-graph is sheeted 2-tangle graph with two vertices.

Case 15 The folding of tangle edge to an edge which is not 1-neighbourhood tangle edge, see Figure 20.

$<$ Figure 20>

The adjacency and incidence matrices of $\mathrm{G}_{t}^{\prime}$ given by

$$
A\left(G_{t}^{\prime}\right)=\left[\begin{array}{cc}
1^{122^{1} 3^{0}} & 1_{1 s^{1}} 1 s^{1} 1 s^{1} \\
1_{1 s^{1} 1 s^{1} 1 s^{1}} & 0
\end{array}\right], I\left(G_{t}^{\prime}\right)=\left[\begin{array}{c}
1_{1 s^{1}}^{1^{1} 2^{1} 3^{0}} s^{1} \\
1_{1 s^{1} 1 s^{1} 1 s^{1}}
\end{array}\right]
$$

Proposition 22 The folding of a tangle-edge to other tangle-edge is a sheeted 2-tangle-graph with loop.

Proposition 23 The limit of foldings of a tangle-edge to other tangle-edge in a sheeted 2-tangle-graph is a sheeted 2tangle-graph with two vertices and loops.

Case 16 Folding of a tangle-edge into itself, see Figure 21.

$<$ Figure 21>

Proposition 24 The limit of foldings of a tangle-edge into itself in a rectangle 2-tangle-graph is a sheeted 2-tangle-graph decrease the number of tangle-vertices and tangle-edge by 1 . 
Proposition 25 The end of limit of foldings of an edge of a rectangle 2-tangle-graph into itself is a 2-tangle-vertex which has the largest number of vertices.

Case 17 Folding of all edges into themselves, see Figure 22.

$<$ Figure 22>

Proposition 26 The limit of foldings of all edges to themselves in a sheeted 2-tangle-graph is a null sheeted 2-tangle-graph has only one tangle vertex.

Proposition 27 The limit of foldings of all tangle-edges into themselves in a rectangle 2-tangle-graph is equivalent to the end of limit of foldings of a tangle-edge into itself.

Case 18 Folding of inner edges into itself, see Figure 23.

$<$ Figure 23>

Proposition 28 The limit of foldings of inner edges into itself of 2-tangle-graph in given a 1-tangle-graph.

Case 19 Folding of each inner vertex into itself, see Figure 24.

$<$ Figure 24>

Proposition 29 The limit of foldings of each inner vertex into itself in a sheeted 2-tangle-graph in disc 2-tangle-graph.

2.3 A general 2-tangle-graph in which each edge is a cylinder and each vertex is a circle

As shown in Figure 25.

$<$ Figure 25>

$$
A\left(G_{t}\right)=\left[\begin{array}{ccc}
0 & 1_{1 c^{2}} & 0 \\
1_{1 c^{2}} & 0 & 1_{1 c^{2} c 1^{2}} \\
0 & 1_{1 c^{2} c 1^{2}} & 0
\end{array}\right], I\left(G_{t}\right)=\left[\begin{array}{cc}
1_{1 c^{2}} & 0 \\
1_{1 c^{2}} & 1_{1 c^{2} c 1^{2}} \\
0 & 1_{1 c^{2} c 1^{2}}
\end{array}\right]
$$

Case 20 The folding of tangle-vertex to its 1-neighbourhood tangle-vertex such folding $V_{1}$ to $V_{2}$ in FIGURE 14 , we obtain a tangle-graph $G_{t}$ in Figure 26.

$<$ Figure 26>

The adjacency and incidence matrices of $G_{t}$ given by

$$
\begin{aligned}
& A\left(G_{t}^{\prime}\right)=\left[\begin{array}{ccc}
1^{1^{0} 2^{2}} & 1_{1_{c^{2}} 1_{c^{2}}} & 0 \\
1_{1_{c^{2}} c_{c^{2}}} & 0 & 1_{1_{c^{2}} 1_{c^{2}} 1_{c^{2}}} \\
0 & 1_{1_{c^{2}} 1_{c^{2}} 1_{c^{2}}} & 0
\end{array}\right], I\left(G_{t}^{\prime}\right)=\left[\begin{array}{cc}
1_{1_{c^{2}} 1_{c^{2}}} 2^{2} & 0 \\
1_{c^{2} 1_{c^{2}}} & 1_{1_{c^{2}} 1_{c^{2}} 1_{c^{2}}} \\
0 & 1_{1_{c^{2}} 1_{c^{2}} 1_{c^{2}}}
\end{array}\right], \\
& A\left(G_{t}^{\prime \prime}\right)=\left[\begin{array}{cc}
1_{1}^{1^{0} 2^{2} 1^{2} 2^{1}} & 1_{c^{2} 1_{c^{2}} 1_{c^{2}}} \\
1_{1_{c^{2}} c^{2} c^{2}} & 0
\end{array}\right], I\left(G_{t}^{\prime \prime}\right)=\left[\begin{array}{c}
1_{1^{2}}^{1^{0} 2^{2} 1^{2} 2^{1}} \\
1_{c^{2} c^{2} 1^{2} c^{2}}
\end{array}\right], \\
& A\left(G_{t}^{\prime \prime \prime}\right)=\left[1^{1^{0} 2^{2} 1^{2} 2^{1} 1^{1} 2^{3} 3^{2}}\right], I\left(G_{t}^{\prime \prime}\right)=[0] .
\end{aligned}
$$

Proposition 30 The graph results from folding of tangle vertex to its 1-neighbourhood tangle vertex in a cylindrical 2-tangle graph is a 2-tangle graph with loop.

Proposition 31 The limit of foldings of 2-tangle vertex into its 1-neighbourhood tangle vertex in a cylindrical 2-tanglegraph is a null 2-tangle graph with loops.

Case 21 The folding of tangle-vertex to anther tangle-vertex which is not 1-neighbourhood to it, see Figure 27.

$<$ Figure 27>

The adjacency and incidence matrices of $G_{t}, G_{t}^{\prime}, G_{t}^{\prime \prime}$ given by

$$
\begin{gathered}
A\left(G_{t}^{\prime}\right)=\left[\begin{array}{ccc}
0 & 1_{1_{c^{2}} 1_{c^{2}}} & 0 \\
1_{1_{c^{2}} 1_{c^{2}}} & 0 & 1_{1_{c^{2}} 1_{c^{2}} 1_{c^{2}}} \\
0 & 1_{1_{c^{2}} 1_{c^{2}} 1_{c^{2}}} & 0
\end{array}\right], I\left(G_{t}^{\prime}\right)=\left[\begin{array}{cc}
1_{1_{c^{2}} 1_{c^{2}}} & 0 \\
1_{1^{2} 1_{c^{2}}} & 1_{1_{c^{2}} 1_{c^{2}} 1_{c^{2}}} \\
0 & 1_{1_{c^{2}} 1_{c^{2}} 1_{c^{2}}}
\end{array}\right], \\
A\left(G_{t}^{\prime \prime}\right)=\left[\begin{array}{ccc}
0 & 1_{1_{c^{2}} 1_{c^{2}}} 1_{1_{c^{2}}} & 0 \\
1_{1_{c^{2}} 1_{c^{2}} 1_{1_{c^{2}}}} & 0 & 1_{1_{c^{2}} 1_{c^{2}} 1_{c^{2}}} \\
0 & 1_{1_{c^{2}} 1_{c^{2}} 1_{c^{2}}} & 0
\end{array}\right],
\end{gathered}
$$




$$
I\left(G_{t}^{\prime \prime}\right)=\left[\begin{array}{ccc}
1_{c^{2}} & 1_{1_{c^{2}} 1_{c^{2}}} & 0 \\
1_{c_{c^{2}}} & 1_{c^{2}} c_{c^{2}} & 1_{1_{c^{2}} 1_{c^{2}} 1_{c^{2}}} \\
0 & 0 & 1_{1_{c^{2}} 1_{c^{2}} 1_{c^{2}}}
\end{array}\right]
$$

Proposition 32 The foldings of tangle-vertex of a cylindrical 2-tangle-graph without loop into another tangle vertex which is not 1-neighbourhood-vertex is a cylinder 2-tangle graph without loop too.

Proposition 33 The limit of foldings of a tangle-vertex to other tangle-vertex which is not 1-neighbourhood vertex to it in a circle 2-tangle-graph is a cylinder 2-tangle-graph with two tangle-vertex.

Case 22 Folding of terminal tangle vertices, see Figure 28.

$<$ Figure 28>

$$
A\left(G_{t}^{\prime}\right)=\left[\begin{array}{ccc}
0 & 1_{c^{2}} & 1_{1_{c^{2}} 1_{c^{2}} 1_{c^{2}}} \\
1_{1_{c^{2}}} & 0 & 1_{c^{2}} c_{c^{2}} \\
1_{c_{c^{2}} 1_{c^{2}} 1_{c^{2}}} & 1_{1_{c^{2}} 1_{c^{2}}} & 0
\end{array}\right], I\left(G_{t}^{\prime}\right)=\left[\begin{array}{ccc}
1_{1_{c^{2}}} & 0 & 1_{1_{c^{2}} 1_{c^{2}} 1_{c^{2}}} \\
1_{1_{c^{2}}} & 1_{c_{c^{2}} 1_{c^{2}}} & 0 \\
0 & 1_{c_{c^{2}} 1_{c^{2}}} & 1_{1_{c^{2}} 1_{c^{2}} 1_{c^{2}}}
\end{array}\right] .
$$

Proposition 33 The foldings of termenal tangle-vertices in open cylinder 2-tangle-graph in a closed cylinder 2-tanglegraph.

case 23 Folding of a tangle-edge to its 1-neighbourhood tangle-edge, see Figure 29.

$<$ Figure 29>

Proposition 34 The foldings of a tangle-edge to its 1-neighbourhood tangle-edge in a cylinder 2-tangle-graph is 2-tanglegraph with the number of edges decrease the tangle-edges in a cylinder 2-tangle-graph by 1 .

Proposition 35 The limit of foldings of a tangle edge into its 1-neighbourhood tangle edge in a cylinder 2-tangle-graph is cylinder 2-tangle graph with two vertices.

Case 24 The folding of a tangle-edge to other tangle-edge which is not 1-neighbourhood tangle-edge, see Figure 30.

$<$ Figure 30>

Proposition 36 The foldings of a tangle-edge to its other tangle-edge in a cylinder 2-tangle-graph without loops is a cylinder 2-tangle-graph with loops.

Case 25 Folding of tangle-edges itself, see Figure 31.

$<$ Figure 31>

Proposition 37 The limit of foldings of a tangle-edge into itself in a cylinder 2-tangle-graph is a cylinder 2-tangle-graph decrease the number of tangle-vertices and tangle-edge by 1 .

Proposition 38 The end of the limit of foldings of an edge of a cylinder 2-tangle-graph into itself is a 2-tangle-vertex which has the largest number of vertices.

Case 26 Folding of tangle-edges into Themselves, see Figure 32.

$<$ Figure 32>

Proposition 39 The limit of foldings of all edges into themselves in a cylinder tangle-graph is a null cylinder tangle-graph has only one tangle vertex.

Proposition 40 The limit of foldings of a tangle-edges into themselves in a cylinder 1-tangle-graph is equivalent to the end of limit of foldings of a tangle-edge into itself.

Case 27 The folding of inner edge into itself, see Figure 33.

$<$ Figure 33>

Proposition 41 The limit of foldings of each inner edges into itself in a cylinder 2-tangle-graph is a linear 1-tangle-graph.

Case 28 Folding of each inner vertex into itself, see Figure 34.

$<$ Figure 34>

Proposition 42 The limit of foldings of each inner vertex into itself in a cylinder 2-tangle-graph is acircle 1-tangle-graph.

\section{References}

A. T. White. (1973). Graph, groups and Surfaces. Amsterdam, North-Holland, Publishing Company. 
Kunio Marasugi, \& Bohdan I. Kurpita. (1999). A study of braids. Kluwer Academic pub. London.

L. W. Beineke, \& R. J. Wilson. (1983). Selected topics in graph theory 2. Academic press Inc. LTD, London.

M. El-Ghoul. (1993). Folding of fuzzy graphs and fuzzy spheres. Fuzzy sets and systems, 58, 355-363, North- Holland. http://dx.doi.org/10.1016/0165-0114(93)90509-G

M. El-Ghoul, \& T. Homoda. (2006). Folding of chaotic multiple graphs and its fractal. International Fuzzy Mathematics Institute, 14(2), USA (In press).

P. J. Giblin. (1977). Graphs, surfaces and homology, an introduction to algebraic topology. Chapman and Hall I, td, London.

R. J. Wilson. (1972). Introduction to graph theory. Oliver \& Boyed, Edinburgh.

R. J. Wilson, \& J. J. Watkins. (1990). Graphs, an introductory approach, a first course in discrete mathematics. Jon Wiley \& Sons Inc, Canada.
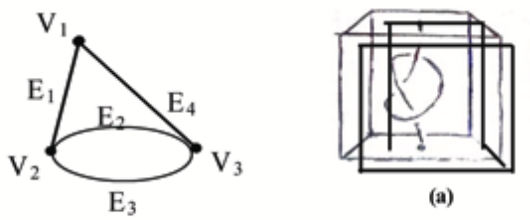

(a)

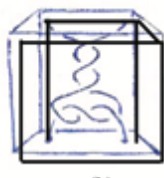

(b)
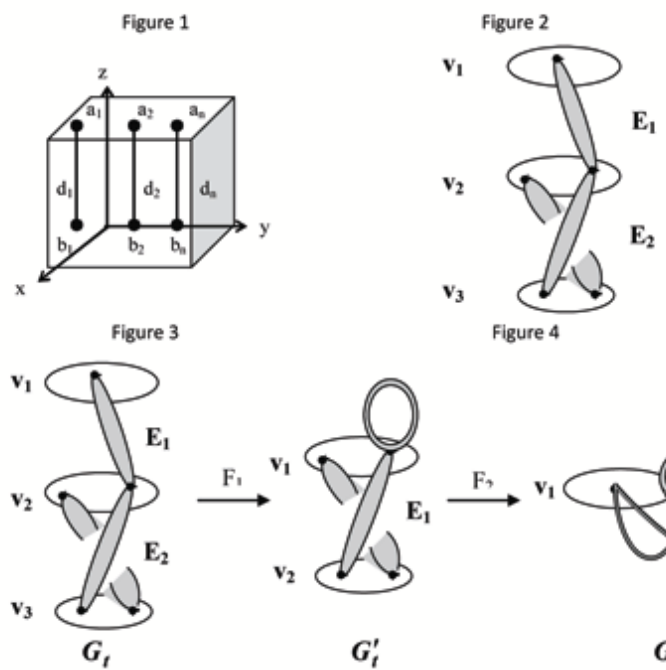

$G_{t}^{\prime}$
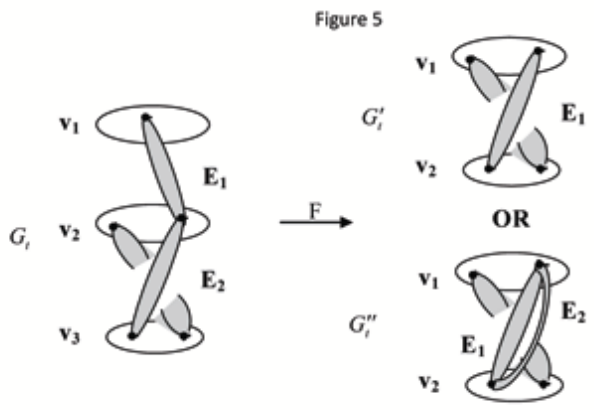

Figure 6 


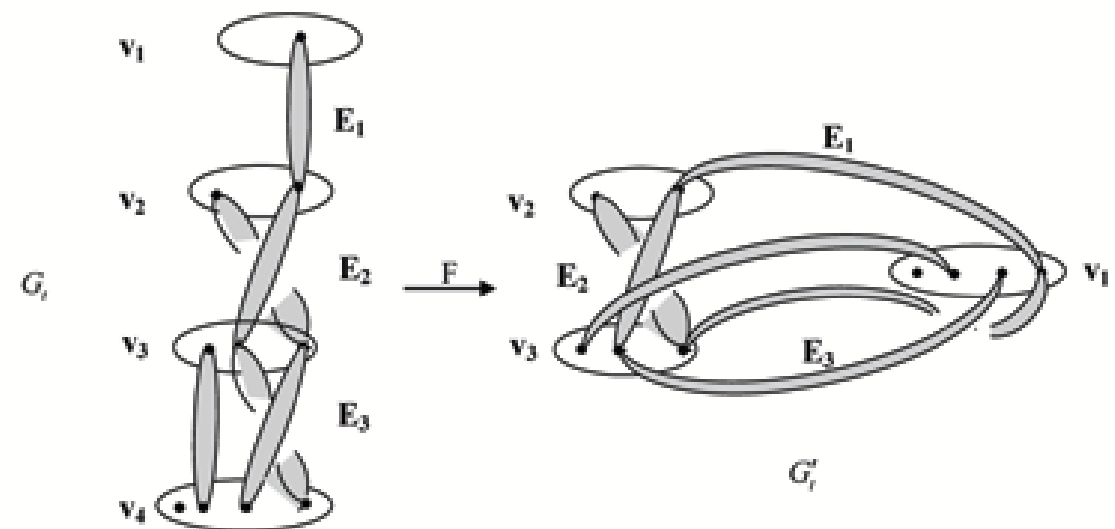

Figure 7

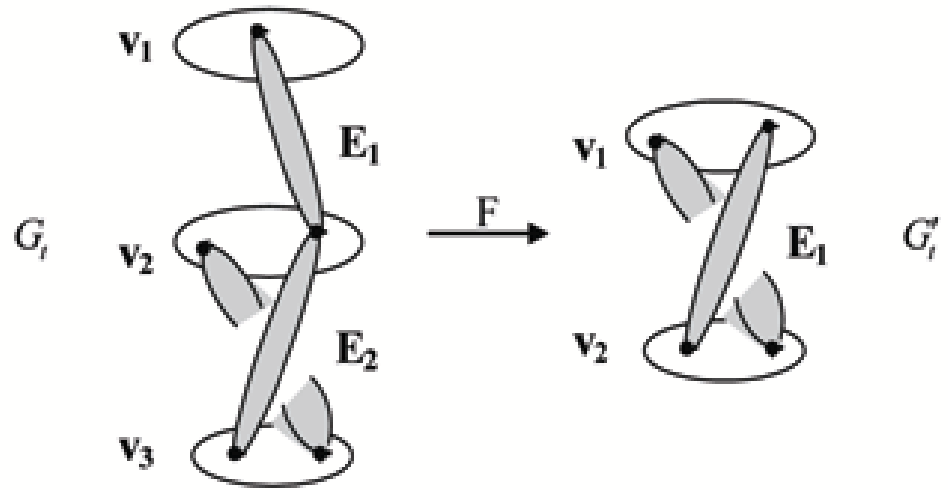

Figure 8

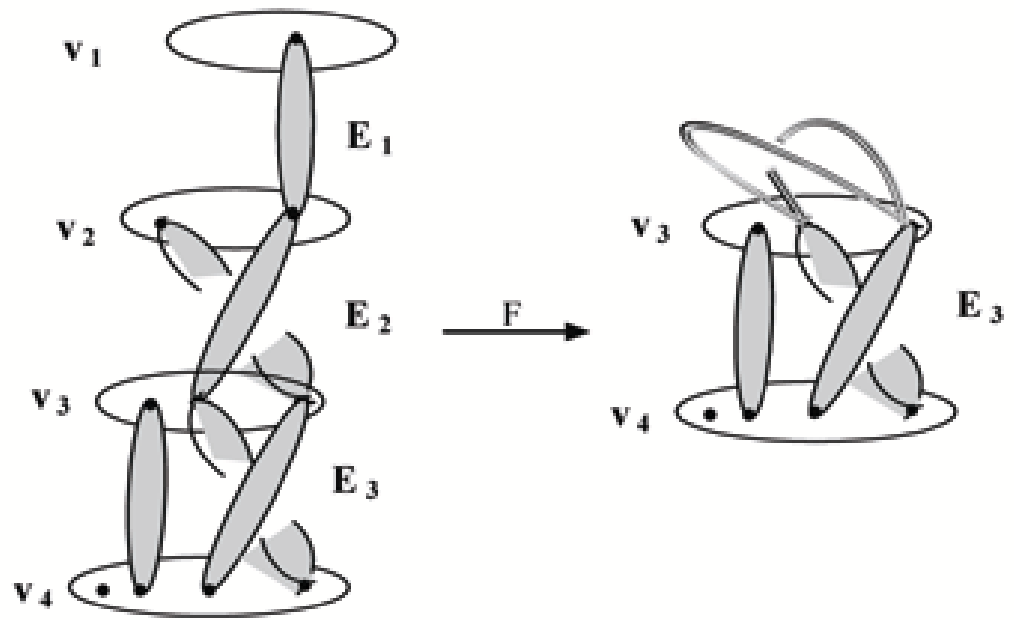

Figure 9 


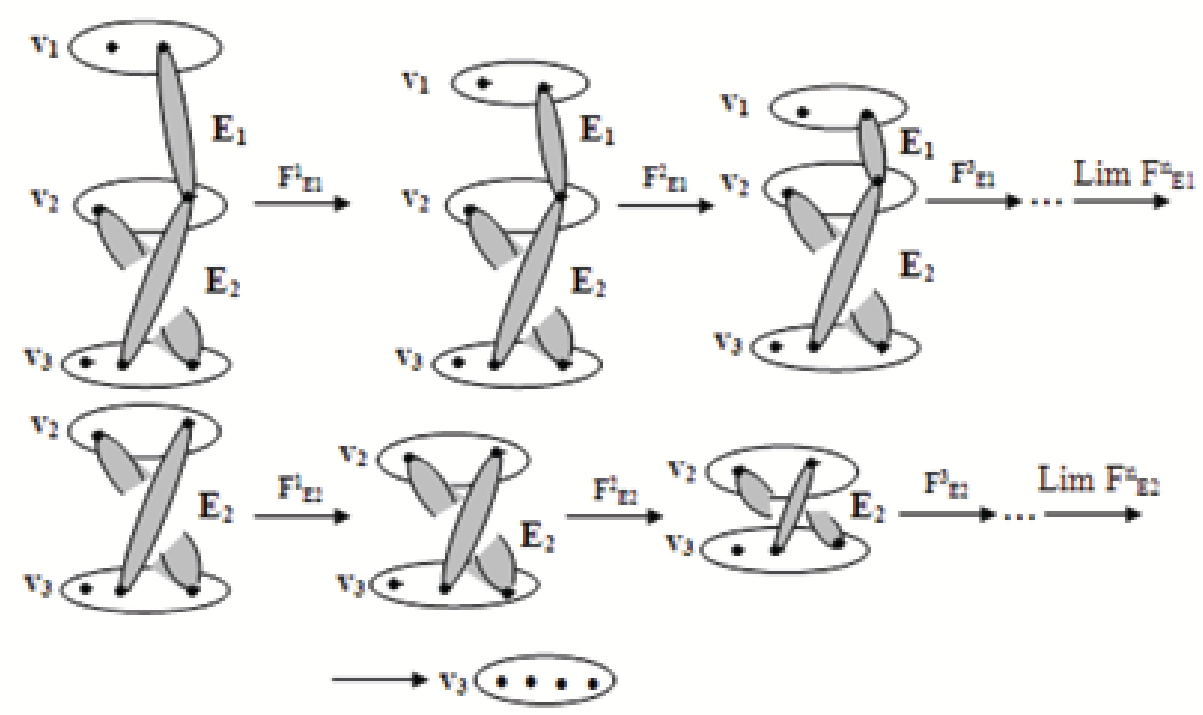

Figure 10
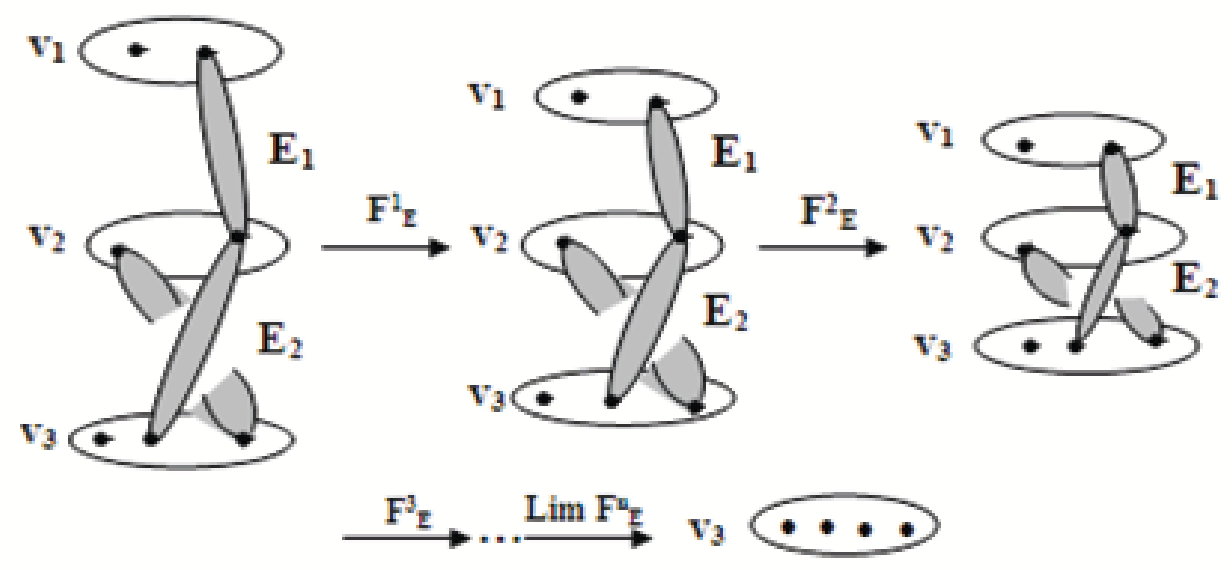

Figure 11
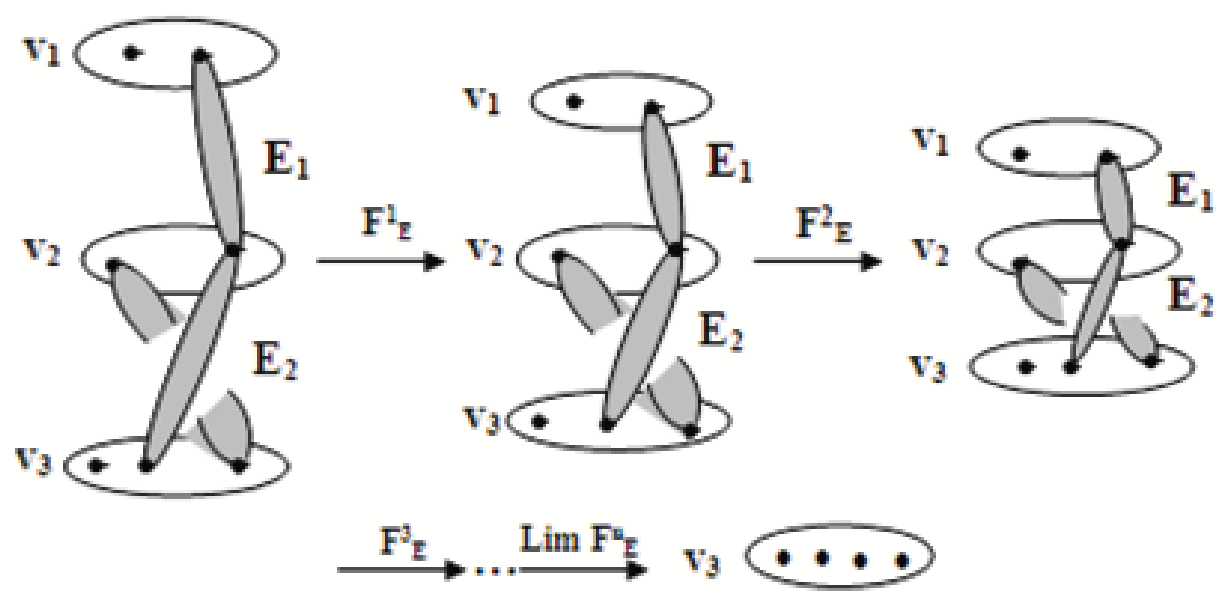

Figure 12 


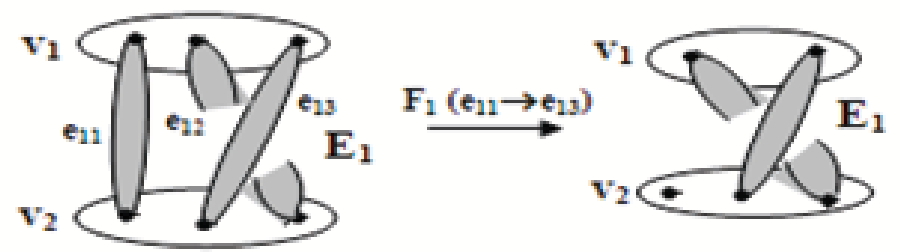

Figure 13

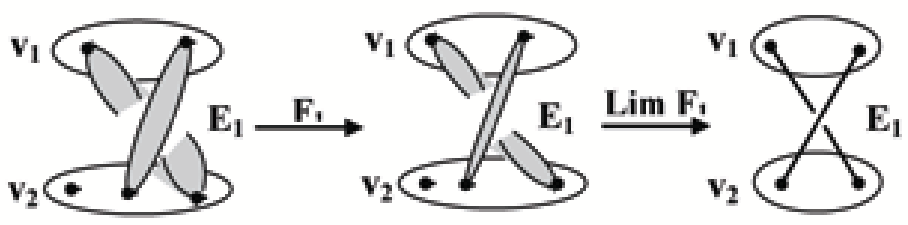

Figure 14

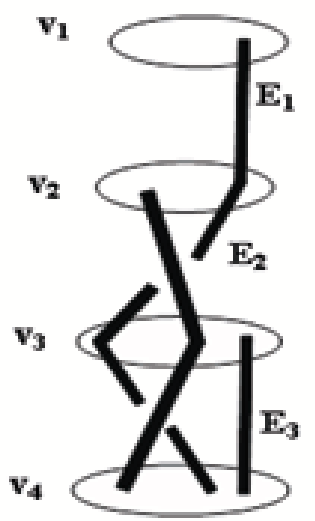

Figure 15
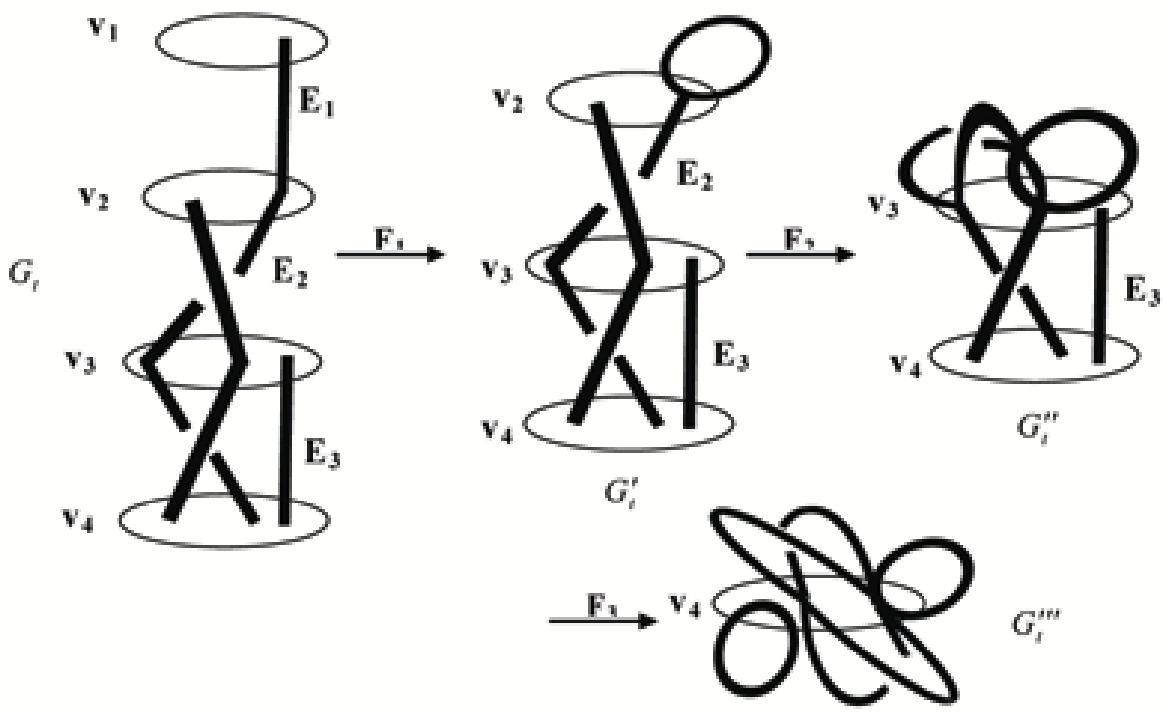

Figure 16 


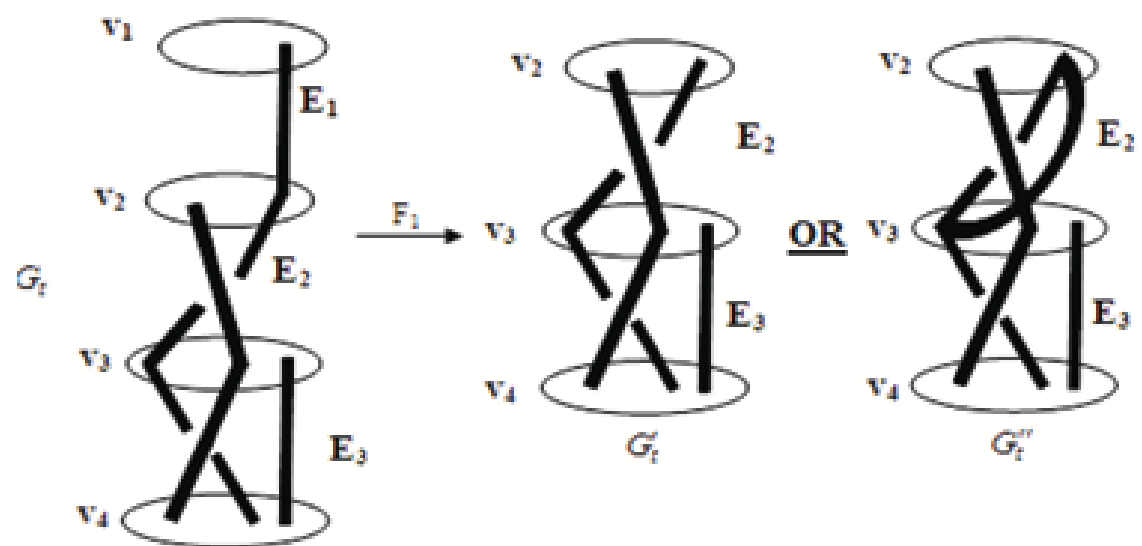

Figure 17

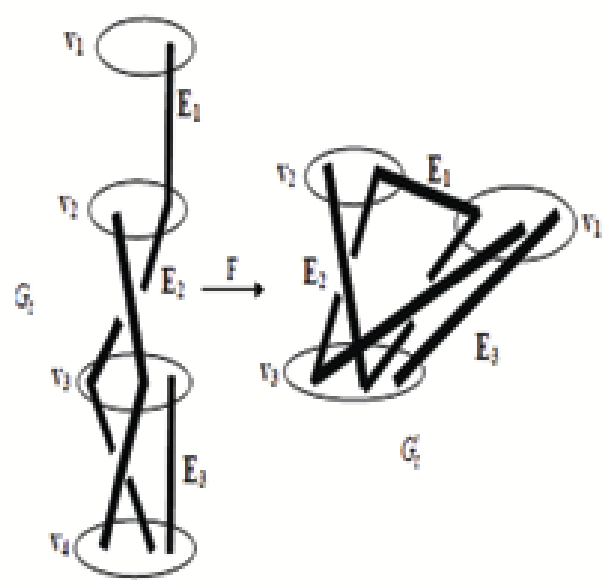

Figure 18

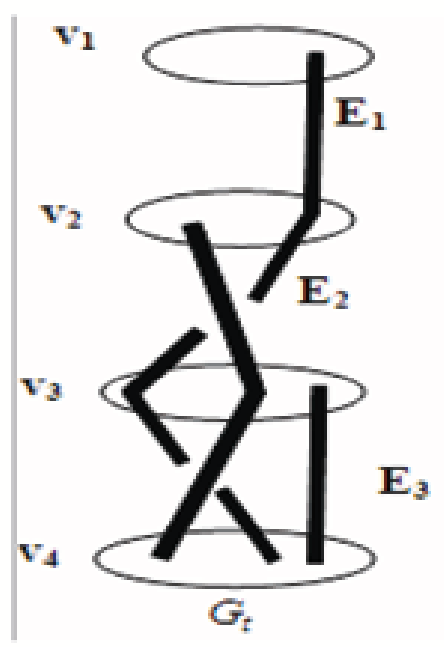

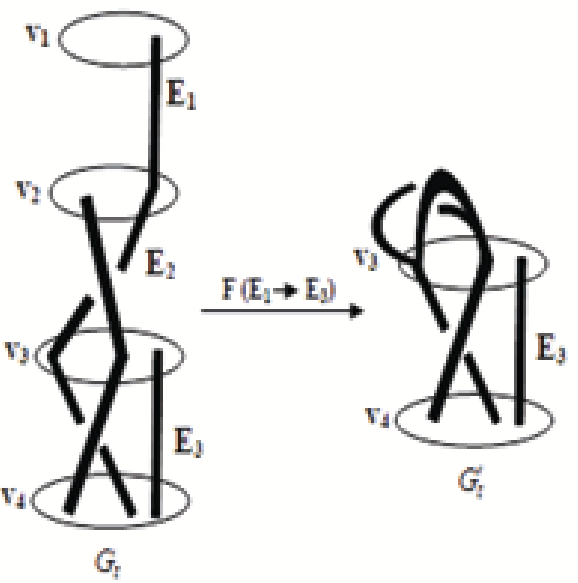

Figure 19

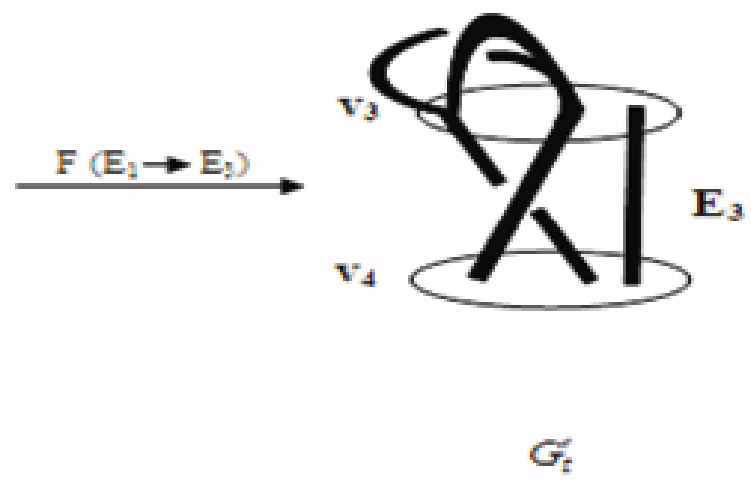

Figure 20 


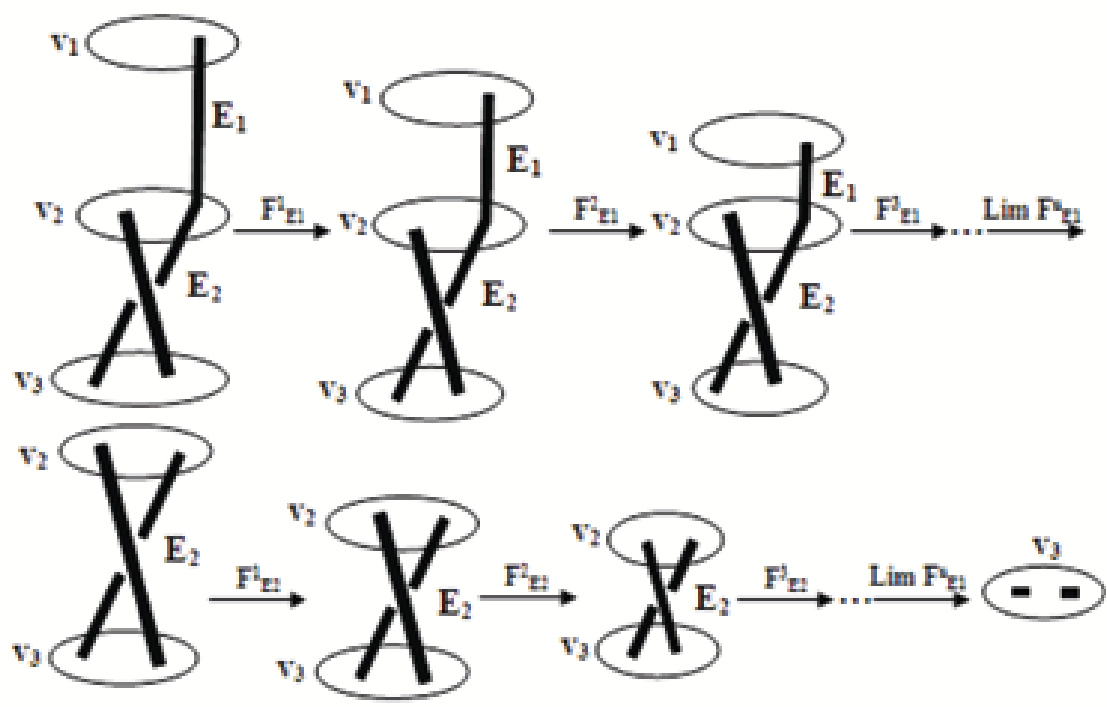

Figure 21

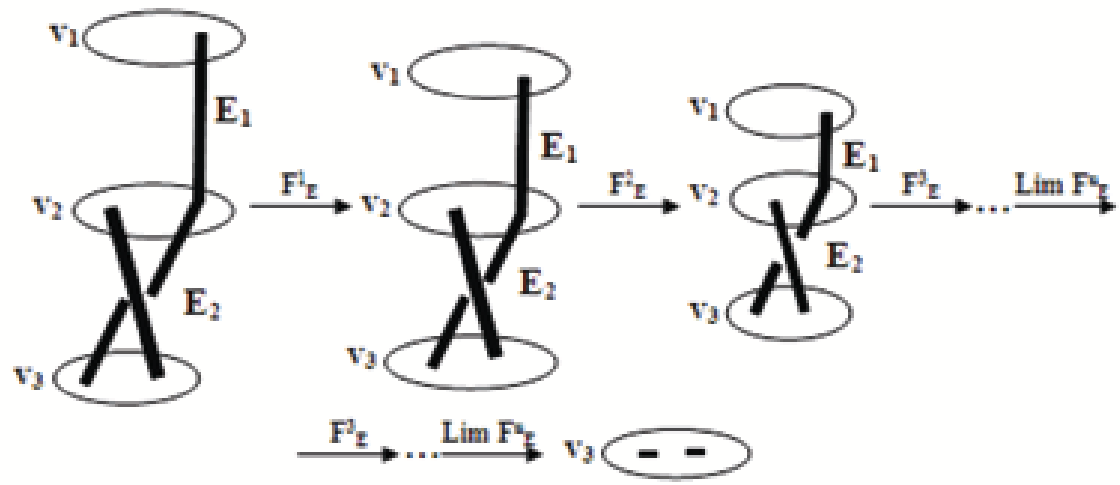

Figure 22

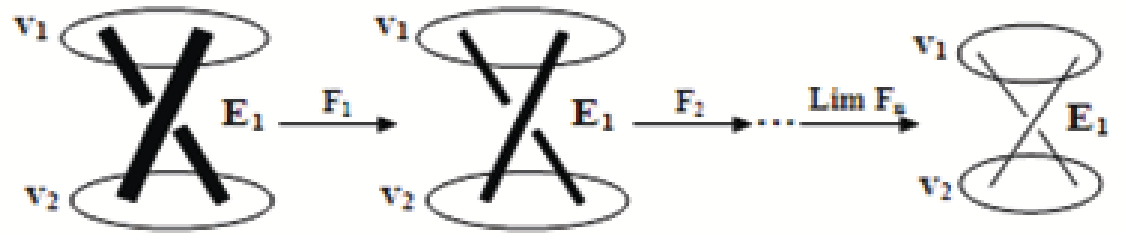

Figure23

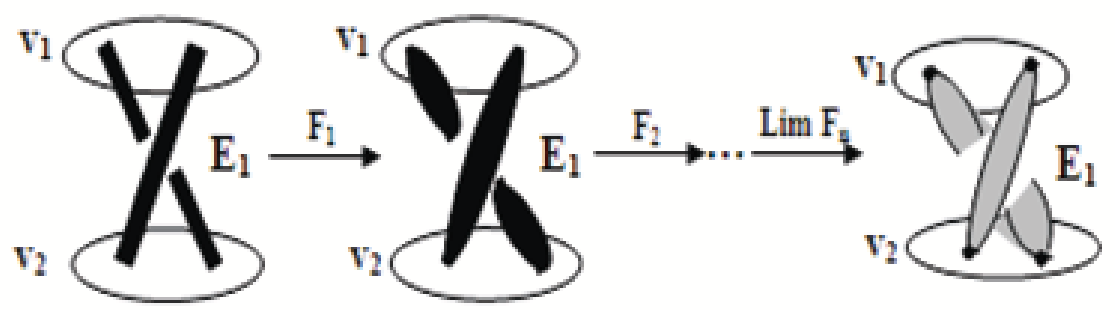

Figure 24 


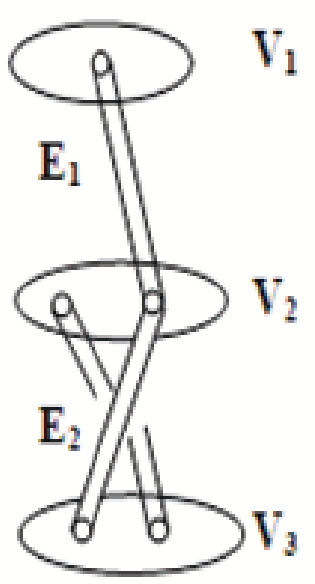

Figure 25

1

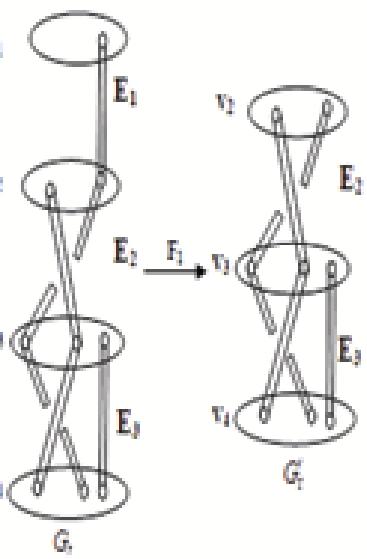

Figure 27

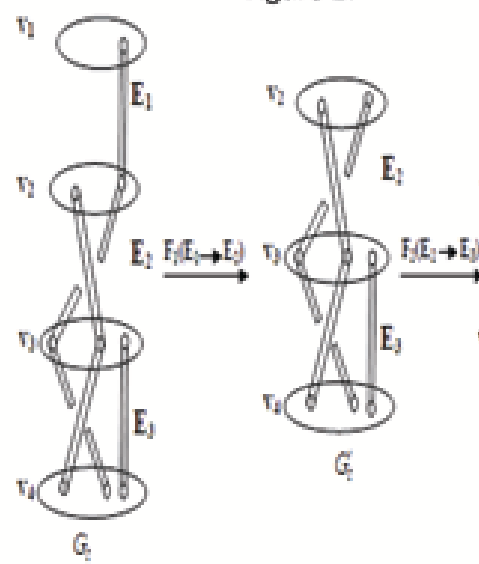

Figure 29

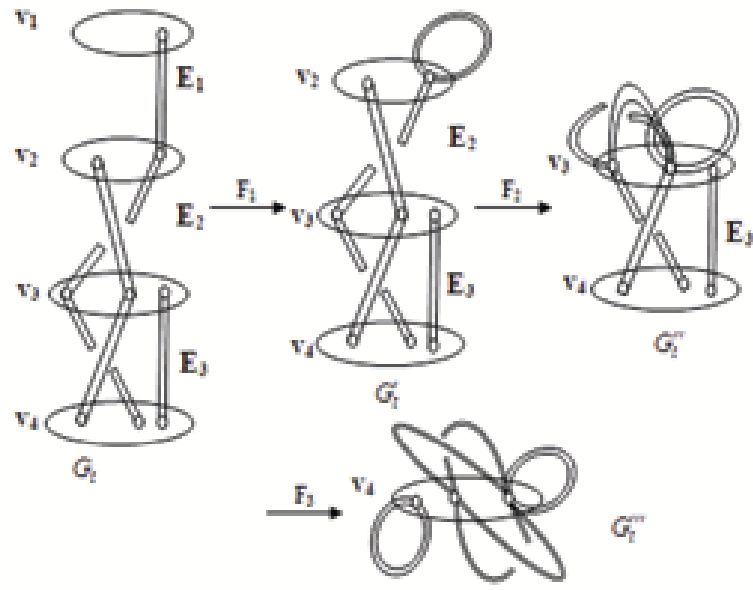

Figure 26

$v_{1}$
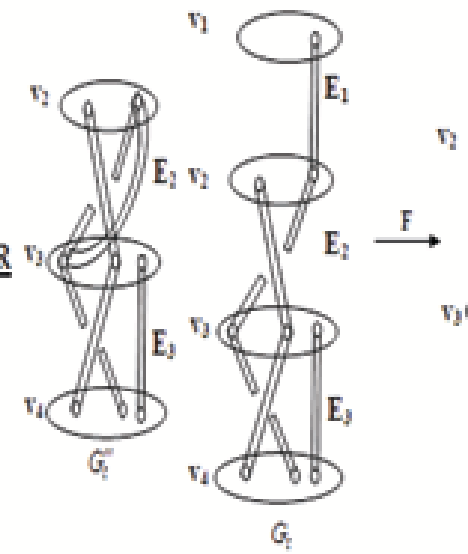

Figure 28

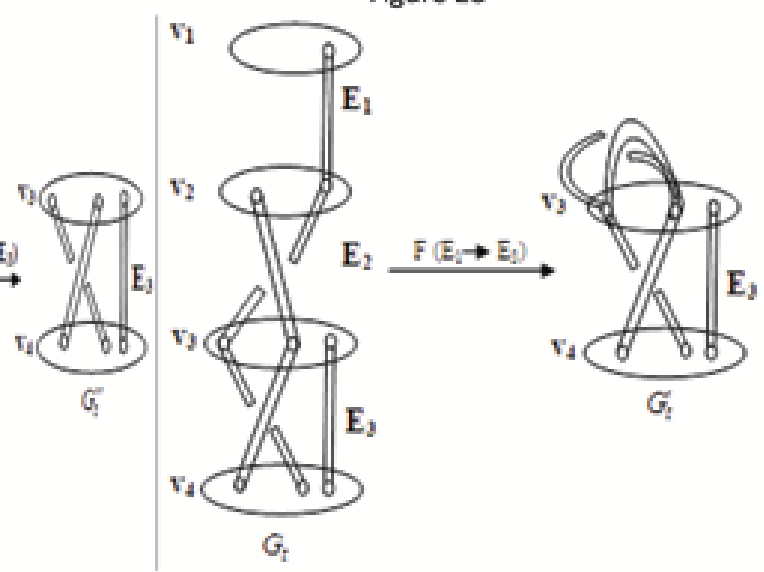

Figure 30 


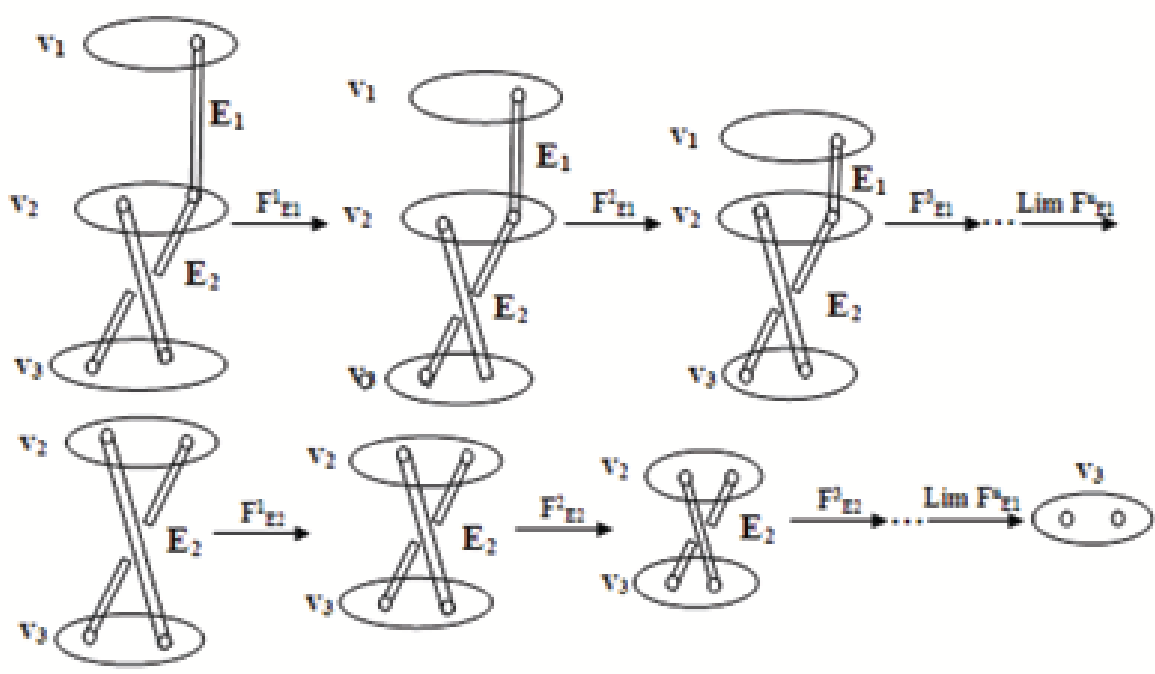

Figure 31

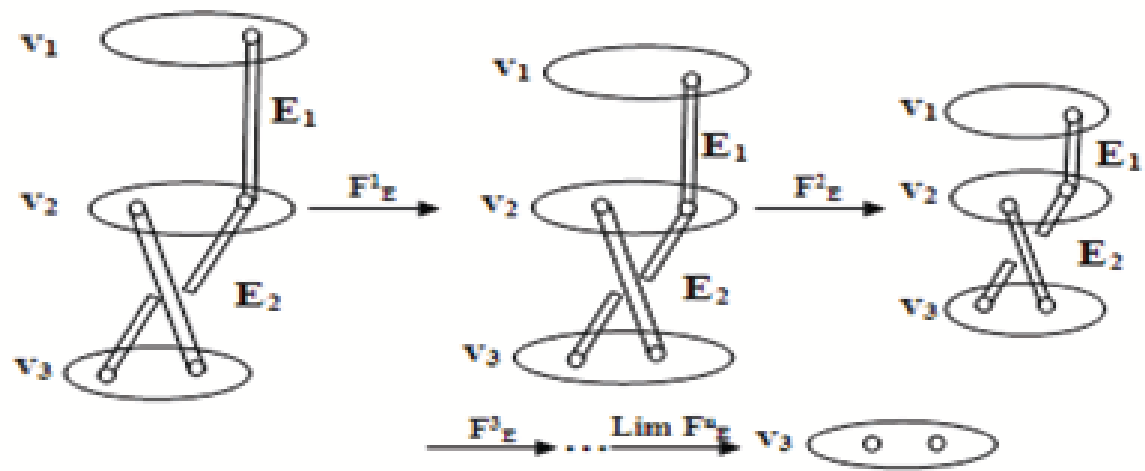

Figure 32

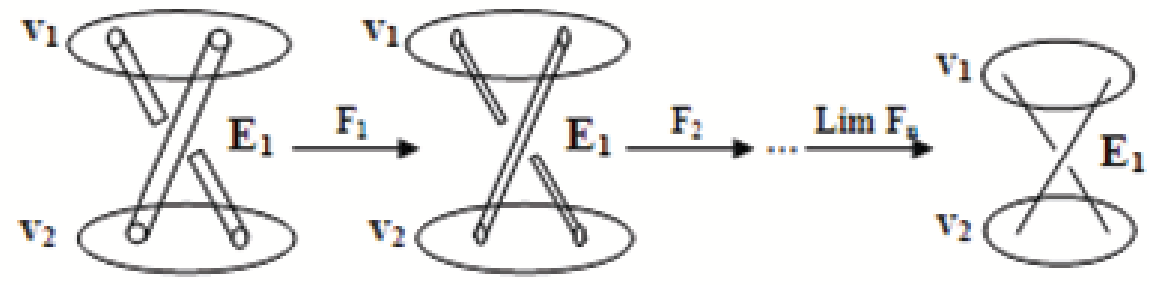

Figure 33

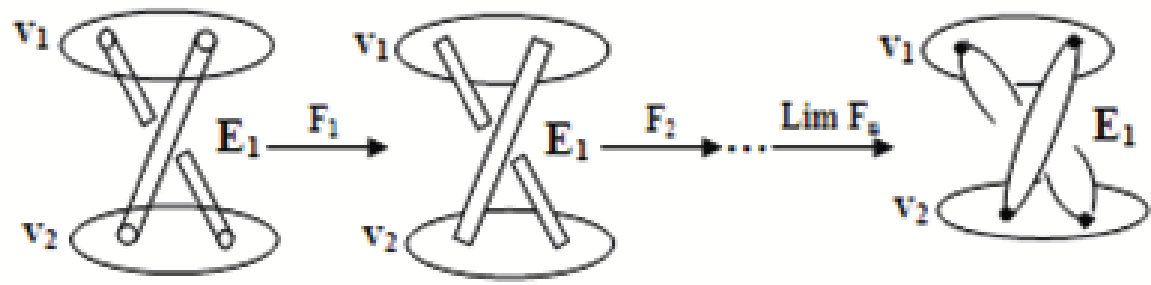

Figure 34 